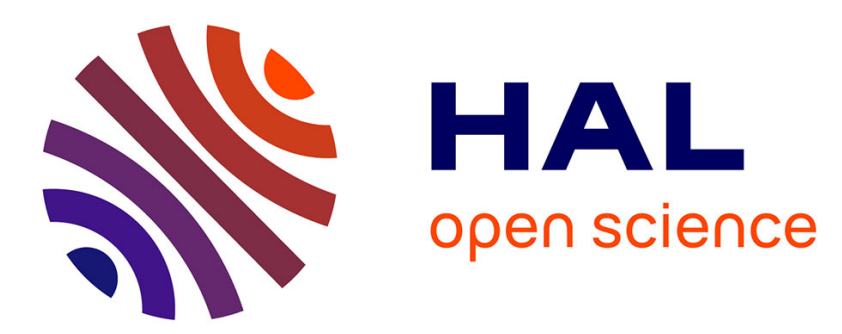

\title{
Time-Reversed Excitation of Reveberation Chambers: Improving Efficiency and Reliability in the Generation of Radiated Stress
}

\author{
Henri Vallon, Andrea Cozza, Florian Monsef, Anne-Sophie Chauchat
}

\section{- To cite this version:}

Henri Vallon, Andrea Cozza, Florian Monsef, Anne-Sophie Chauchat. Time-Reversed Excitation of Reveberation Chambers: Improving Efficiency and Reliability in the Generation of Radiated Stress. IEEE Transactions on Electromagnetic Compatibility, 2016, 99 (00), 10.1109/TEMC.2015.2478420 . hal-01197059

\author{
HAL Id: hal-01197059 \\ https://hal.science/hal-01197059
}

Submitted on 11 Sep 2015

HAL is a multi-disciplinary open access archive for the deposit and dissemination of scientific research documents, whether they are published or not. The documents may come from teaching and research institutions in France or abroad, or from public or private research centers.
L'archive ouverte pluridisciplinaire HAL, est destinée au dépôt et à la diffusion de documents scientifiques de niveau recherche, publiés ou non, émanant des établissements d'enseignement et de recherche français ou étrangers, des laboratoires publics ou privés. 


\title{
Time-Reversed Excitation of Reveberation Chambers: Improving Efficiency and Reliability in the Generation of Radiated Stress
}

\author{
Henri Vallon, Andrea Cozza, Florian Monsef, Anne-Sophie Chauchat
}

\begin{abstract}
The ability of reverberation chambers to generate high-intensity field levels from relatively low-power input signals is reexamined for the case of time-reversed signals, proving that they lead to a higher efficiency. Moreover, the strong statistical spread typical of time-harmonic excitations can be dramatically reduced, thus improving the reliability of radiative tests, while limiting the need for a large number of independent realizations. The two excitation schemes are compared when forcing their respective input signals to display the same peak instantaneous power. Experimental results are provided, supporting the conclusions of our theoretical analysis.
\end{abstract}

Index Terms-Conversion efficiency, radiated test, reverberation chamber, time reversal, statistical electromagnetics

\section{INTRODUCTION}

$\mathbf{R}$ EVERBERATION chambers (RCs) are capable of converting harmonic signals into high-intensity fields, by creating a pool of stored electromagnetic energy, thanks to their relatively long relaxation time constants. The stored energy can reasonably be expected to be uniformly spread over the entire volume of the RC, thus allowing for an ideally global excitation of an equipment under test (EUT).

Yet, this property also implies that, at each instant, only a portion of the energy injected into the $\mathrm{RC}$ interacts with the EUT. This observation is the starting point of our proposal for using time reversal (TR) as an alternative to harmonic or continuous-wave $(\mathrm{CW})$ excitations. TR allows the generation of wavefronts focusing both in space and time; in other words, it allows concentrating a large portion of energy over a region of space at a given time. Studies of applications of TR to cavities are not new [1]-[3], but they have been mainly concerned with demonstrating the possibility of producing focusing signals while propagating through non-anechoic media. We are rather interested in assessing how much TR can increase the peak intensity of the power used to stress an EUT.

This paper addresses this topic by analyzing the conversion efficiency of an RC when using TR signals rather than standard harmonic ones. The problem is tackled from a theoretical point of view, with the aim of providing simple predictive tools for the design of TR excitations. Our results prove that the benefits of TR come in three shapes: 1) ensuring a higher

A. Cozza and F. Monsef are with PIEM, GeePs, UMR 8507, CentraleSupelec, Univ Paris-Sud, UPMC, CNRS, 1 rue Joliot-Curie, 91192 Gif-surYvette, France. A.-S. Chauchat is with THALES Communications \& Security Gennevillers. H. Vallon is working with both institutions. Part of the work here reported was funded by the French National Research Agency through the grant ANR-12-ASTR-0005-01, MIMOCHIC project, within the framework of the 2012 ASTRID program. Contact e-mail: andrea.cozza@ieee.org field intensity, 2) reducing the uncertainty of the actual level of the generated field and 3) reducing the need for multiple realizations (i.e., stirring iterations) for the sake of producing a higher stress on the EUT. Even though not discussed in this paper, a fourth advantage should also be mentioned, namely the possibility of controlling the polarization of the resulting field, as proven in [4]. The comparisons are carried out by acknowledging a fundamental limitation of power amplifiers, namely the maximum peak instantaneous power (PIP) they can handle. This constraint calls for an analysis differing from that presented in [5], while also taking into account the highly fluctuating behavior of RC-generated signals.

Only point-like focusing is considered in this paper, which means focal spots with spatial extensions subject to the diffraction limit; in practice, about half a wavelength wide. Extension to other kinds of wavefronts is possible by using generalized TR, a technique described in [6]. This same reference also discusses practical issues, e.g., how wavefronts are generated and moved around an EUT. This paper will only focus on how using TR excitations can improve the generation of electromagnetic stresses within an RC.

\section{CONVERSION EFFICIENCIES}

The point of view adopted in this paper is that an $\mathrm{RC}$ is a linear system that converts an input signal $x(t)$, say a voltage, applied at the input port of an excitation antenna, into a vector electric field $\boldsymbol{e}(\boldsymbol{r}, t)$ measured within the RC at the position $\boldsymbol{r}$. In the rest of this paper we will drop the variable $r$, for the sake of brevity, and derive results that are valid for any $\boldsymbol{r}$ within the test volume, where we expect a diffuse-field approximation to hold. The average composite quality factor $Q$ of the RC will be considered to be a good estimate of the composite quality factors experienced over the bandwidth used, around a working frequency $f_{c}$. The average time constant of electromagnetic fields in the RC will thus be defined as $\tau=Q / \pi f_{c}$.

The field thus generated is expected to stress a portion of a circuit in an EUT, which will "receive" the power $p_{r}(t)$ as a linearly-polarized antenna, i.e.,

$$
p_{r}(t)=C_{r}[\boldsymbol{e}(t) \cdot \hat{\boldsymbol{q}}]^{2}=C_{r} f^{2}(t)
$$

where $C_{r}$ represents the sensitivity of the receiver, assumed to be non-dispersive, with no loss of generality, while $\hat{\boldsymbol{q}}$ is the polarization-vector of the receiver. Similarly, we consider the input available power $p_{i}(t)=C_{i} x^{2}(t)$ applied at the antenna input port, with $C_{i}$ a constant of units equal to a conductance. 
In view of the generation of a radiated stress, the conversion efficiency

$$
\eta_{\mathrm{S}}=\eta\left[x_{S}(t)\right]=\frac{\left\|p_{r}(t)\right\|_{\infty}}{\left\|p_{i}(t)\right\|_{\infty}}=\frac{C_{r}}{C_{i}} \frac{\left\|f_{S}^{2}(t)\right\|_{\infty}}{\left\|x_{S}^{2}(t)\right\|_{\infty}}
$$

should be maximized, where the subscript $S$ is used in order to distinguish different excitation signals. The rationale for choosing the $L_{\infty}$ norm is twofold: the output PIP stressing the EUT should be as high as possible, while being generated by an input signal with the lowest PIP, in order to avoid any strain on power amplifiers and subsequent non-linear distortions or potential thermal stress.

The $\hat{\boldsymbol{q}}$-aligned component of the electric field, $f(t)$, is linearly related to the excitation signal through a transfer function $\Phi(\nu)$, such that $f(t)=\mathscr{F}^{-1}\{\Phi(\nu) X(\nu)\}(t)$, where $\mathscr{F}^{-1}\{\cdot\}$ is the inverse Fourier transform between the frequency variable $\nu$ and the time variable $t$. The function $X(\nu)$ is the Fourier spectrum of the excitation signal. The following derivations will assume excitation signals with spectra centered at the frequency $f_{c}$, covering the bandwidth $B_{T}=f_{2}-f_{1}$.

Harmonic signals are routinely used in standard operation of RC. In this case $x_{\mathrm{CW}}(t)=A \cos \left(2 \pi f_{c} t\right)$ and thus the conversion efficiency is

$$
\eta_{\mathrm{CW}}=\frac{C_{r}}{C_{i}}\left|\Phi\left(f_{c}\right)\right|^{2}=\frac{C_{r}}{C_{i}} W\left(f_{c}\right),
$$

where $W(\nu)=|\Phi(\nu)|^{2}$ is a short-hand notation. In practice, the response of RCs is randomized by means of stirring techniques; as a result several realizations of $\Phi(\nu)$ can be generated and the resulting efficiency can take very different values. This point will be discussed in sec. III.

In the case of time-reversed excitations, the input signal is chosen to be

$$
x_{\mathrm{TR}}(t)=g(t) * \phi(-t)=\mathscr{F}^{-1}\left\{G(\nu) \Phi^{\star}(\nu)\right\}(t),
$$

where $G(\nu)$ is the Fourier spectrum of the signal $g(t)$ chosen to stress the receiver; $*$ stands for the convolution operator applied to the time variable, while the superscript $\star$ represents the complex conjugate. Any kind of signal can be chosen for $g(t)$, but time reversal has been demonstrated mainly with short pulses; a by-product of our derivation is that indeed better efficiencies are obtained by choosing a $g(t)$ with the most compact support. Therefore, EUTs with a relaxation time longer than that of the cavity, typically in the microsecond range, are not suited to this technique, as their bandwidths would not cover enough degrees of freedom, as explained in the rest of the paper. This limitation can be overcome by using trains of short pulses; this kind of signals does not appear to have been considered so far in the literature and deserve investigations on their own as a practical solution.

Since the electric field $f(t)$ at the receiver position will have a spectrum $|\Phi(\nu)|^{2} G(\nu)$, if $g(t)$ presents a maximum intensity at $t=0$, the same will occur for $f(t)$, given that $|\Phi(\nu)|^{2}$ is real-positive and corresponds to the spectrum of the auto-correlation function of $\phi(t)$, i.e., with its maximum at $t=0$. Hence,

$$
\left\|p_{r}(t)\right\|_{\infty}=C_{r}\left[\int_{B_{T}} \mathrm{~d} \nu G(\nu) W(\nu)\right]^{2}=C_{r} \mathscr{I}^{2} .
$$

Solving the integral $\mathscr{I}$ by parts yields

$$
\mathscr{I}=G\left(f_{2}\right) C_{W}\left(f_{2}\right)-\int_{B_{T}} \mathrm{~d} \nu G^{\prime}(\nu) C_{W}(\nu)
$$

with $G^{\prime}(\nu)$ the first derivative of $G(\nu)$ with respect to $\nu$ and

$$
C_{W}(\nu)=\int_{f_{1}}^{\nu} \mathrm{d} s W(s) \simeq\left(\nu-f_{1}\right) \bar{W}
$$

a primitive of $W(\nu)$. The linear approximation in (7) assumes an average value $\bar{W}$ of $W$ constant over $B_{T}$; indeed, typical RCs present slowly-varying statistical moments for the scalar components of the electric field, as shown in sec. IV. This approximation holds for $\nu-f_{1} \gg B_{c}$, where the $B_{c}=1 / \tau$ is the coherence bandwidth of $\Phi(\nu)$ : in other words, as soon as the integration is carried out over enough significantly different portions of $W(\nu)$. Making use of this approximation in (6) and solving by parts the integral therein yields

$$
\mathscr{I}=\bar{W} \int_{B_{T}} \mathrm{~d} \nu G(\nu)=\bar{W} \bar{G} B_{T}
$$

and finally substituting it into (5) gives

$$
\left\|p_{r}(t)\right\|_{\infty}=C_{r} \bar{W} \bar{G} B_{T} .
$$

This result holds for receivers modeled as in (1), i.e., presenting an flat frequency response over $B_{T}$. An eventual constant group delay could be included, but is not explicited in (1) as it would not affect the PIP. When dealing with dispersive responses, (9) should be modified by weighting $W(\nu)$ by the transfer function of the receiver, before computing its average value over $B_{T}$. Depending on its degree of frequency dispersion, this latter could reduce the frequency average and thus the peak received power. As thus, the case of a flat response must be regarded as a best case for TR excitations.

Before computing $\left\|p_{i}(t)\right\|_{\infty}$, we need to recall that TR makes sense only for impulse-like signals $g(t)$ covering a bandwidth $B_{T} \gg B_{c}$, i.e., for $B_{T} / f_{c} \gg \pi / Q$; under these conditions, $g(t)$ has a time support $T_{g} \ll \tau$, implying that the macroscopic features of $\phi(-t) \star g(t)$ are dominated by those of $\phi(t)$.

Impulse responses in an RC can be modeled as random processes, with a root-mean-square (rms) envelope following $\left\langle|\phi(t)|^{2}\right\rangle=A_{o}^{2} \exp (-2 t / \tau)[7$, ch. 3] [8]. The local deviations of the peak power of these kind of signals with respect to their rms envelope can be expressed as an overshoot factor

$$
K=\sqrt{\left\|p_{i}(t)\right\|_{\infty}} / A_{o},
$$

whose cumulative distribution function is

$$
F_{K}(x)=\prod_{k=0}^{N} \operatorname{erf}\left(x \mathrm{e}^{k / B_{T} \tau} / \sqrt{2}\right)
$$

with $N \geq 4 B_{T} \tau \ln 2$, as demonstrated in [8]. It is now sufficient to express $A_{o}$ as a function of $W(\nu)$; since $A_{o}^{2}=$ $\left\langle x_{\mathrm{TR}}^{2}(0)\right\rangle$,

$$
\left\langle x_{\mathrm{TR}}^{2}(0)\right\rangle=\int_{B_{T}} \int_{B_{T}} \mathrm{~d} \nu_{1} \mathrm{~d} \nu_{2} G\left(\nu_{1}\right) G^{\star}\left(\nu_{2}\right)\left\langle\Phi\left(\nu_{1}\right) \Phi^{\star}\left(\nu_{2}\right)\right\rangle,
$$


where

$$
\left\langle\Phi\left(\nu_{1}\right) \Phi^{\star}\left(\nu_{2}\right)\right\rangle=\left\langle\left|\Phi\left(f_{c}\right)\right|^{2}\right\rangle \mu\left(\nu_{2}-\nu_{1}\right)
$$

is the frequency covariance function, which can be factorized into a slowly-varying power spectral density $\left\langle|\Phi(\nu)|^{2}\right\rangle=$ $\langle W(\nu)\rangle$, here assumed to be constant over $B_{T}$, such that $\langle W(\nu)\rangle \simeq \bar{W}$, and a frequency-coherence function $\mu\left(\nu_{2}-\nu_{1}\right)$ with a much narrower support, of the order of the modal bandwidth of the RC, as discussed in [9].

For any signal $g(t)$ such that its spectrum varies over scales much larger than $B_{c}$, the coherence function operates as a sifting function

$$
\begin{aligned}
& \int_{B_{T}} \mathrm{~d} \nu_{2} G^{\star}\left(\nu_{2}\right) \mu\left(\nu_{2}-\nu_{1}\right) \\
& \simeq G^{\star}\left(\nu_{1}\right) \int_{B_{T}} \mathrm{~d} \nu_{2} \mu\left(\nu_{2}-\nu_{1}\right)=G^{\star}\left(\nu_{1}\right) B_{c},
\end{aligned}
$$

yielding

$$
A_{o}^{2}=\left\langle x_{\mathrm{TR}}^{2}(0)\right\rangle=\bar{W} B_{c} \int_{B_{T}} \mathrm{~d} \nu|G(\nu)|^{2},
$$

where the last integral represents the energy of $g(t)$, in the mathematical sense. Using (9) and (15), the conversion efficiency of time-reversed excitations is

$$
\eta_{\mathrm{TR}}=\frac{C_{r}}{C_{i}} \frac{B_{T}}{B_{c}} \frac{\bar{W} \kappa^{2}}{K^{2}}
$$

with

$$
\kappa^{2}=B_{T} \bar{G}^{2}\left(\int_{B_{T}} \mathrm{~d} \nu|G(\nu)|^{2}\right)^{-1} \leq 1
$$

a shape factor attaining its maximum value for flat Fourier spectra.

\section{POWER GAIN}

How do $\eta_{\mathrm{CW}}$ and $\eta_{\mathrm{TR}}$ compare? In order to answer this question, two points should be considered: the random nature of $W(\nu)$ and the fact that, in case of harmonic excitations, an $\mathrm{RC}$ is typically operated jointly with a stirring technique. The assumption of an overmoded RC, as defined in [10], implies that $W(\nu)$ is bound to follow an exponential probability law [11], [12]. As a result, $\eta_{\mathrm{CW}}$, when computed for a single realization, is widely dispersed around its mean value: e.g., $\eta_{\mathrm{CW}} /\left\langle\eta_{\mathrm{CW}}\right\rangle \in(0.05,3.00)$ with $90 \%$ probability. The poor predictability of the actual stress generated by an RC is improved by having $N$ realizations, introducing stirring techniques [13]. This pragmatic approach is acknowledged by introducing

$$
\eta_{\mathrm{CW}}^{N}=\max _{i \in[1, N]} \eta_{\mathrm{CW}}^{(i)}
$$

i.e., the performance of the harmonic excitation will be evaluated as the highest one out of $N$ realizations. As a result, $x=\eta_{\mathrm{CW}}^{N} /\left\langle\eta_{\mathrm{CW}}\right\rangle$ will follow the probability law [14], [15]

$$
p_{N}(x)=N\left(1-\mathrm{e}^{-x}\right)^{N-1} \mathrm{e}^{-x} .
$$

The strong skewness of this family of probability laws (see Fig. 1) implies that the statistical mode should be regarded as a more accurate estimator of typical values taken by $\eta_{\mathrm{CW}}^{N}$,

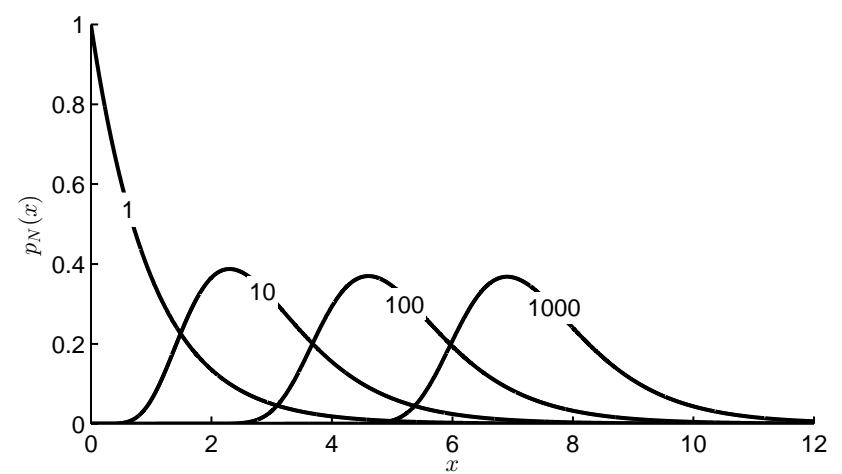

Figure 1. Probability distributions of $\eta_{\mathrm{CW}}^{N} /\left\langle\eta_{\mathrm{CW}}\right\rangle$ for a varying number $N$ of realizations.

instead of its mean value. Therefore, the two conversion efficiencies will be compared by taking the ratio of their modes, a quantity hereafter referred to as the power gain

$$
G_{p}^{N}=\frac{\operatorname{Mo}\left[\eta_{\mathrm{TR}}\right]}{\operatorname{Mo}\left[\eta_{\mathrm{CW}}^{N}\right]},
$$

with $\mathrm{Mo}[\cdot]$ the mode of a population of samples. The rationale for naming this quantity power gain is to be found in the possibility of obtaining the same order of magnitude of stress onto the EUT, while using a lower PIP at the input of the RC, i.e., at the output of the power amplifier. This observation is a direct consequence of having defined the conversion efficiency (2) as a quantity normalized to a fixed peak instantaneous input power.

As opposed to the case of harmonic excitation, time-reversal excitation is characterized by a self-averaging property [16], as long as $B_{T} \gg B_{c}$. This property is apparent in the definition of $C_{W}(\nu)$ in (7), where the integral is taken over a stationary random process $W(\nu)$ which takes only positive real values. The low coherence of $W(\nu)$ outside sub-bandwidths of the order of $B_{c}$ implies that the integral can be recast as a discrete sum

$$
C_{W}(\nu) \simeq B_{c} \sum_{i=1}^{N_{\nu}} W\left(s_{i}\right)
$$

with $s_{i}-s_{i-1}=B_{c}$ and $\nu-f_{1}=N_{\nu} B_{c}$. The stationarity of $W(\nu)$ over $B_{T}$ here translates into i.i.d. $W\left(s_{i}\right)$; hence, the central-limit theorem allows stating that asymptotically $C_{W}$, and ultimately $\left\|f_{\mathrm{TR}}(t)\right\|_{\infty}$, follow a normal distribution with relative standard deviation approximated by $\sqrt{B_{c} / B_{T}}$. This property is confirmed in sec. IV.

Use of a sufficiently large bandwidth can therefore be expected to reduce the uncertainty in the TR-generated field; this key point is discussed in secs. IV and V. Convergence to a weakly fluctuating $\left\|f_{\mathrm{TR}}(t)\right\|_{\infty}$ invites the following approximation: to consider that the randomness of $\eta_{\mathrm{TR}}$ is only due to $\left\|x_{\mathrm{TR}}^{2}(t)\right\|_{\infty}$. Under this assumption, the pdf of $\eta_{\mathrm{TR}}$ can be expressed as a function of the pdf of the random variable $1 / K^{2}$, as apparent from (16)

$$
p_{K^{-2}}(x)=\frac{(x / \alpha)^{-3 / 2}}{2 \alpha} p_{K}(\sqrt{\alpha / K})
$$




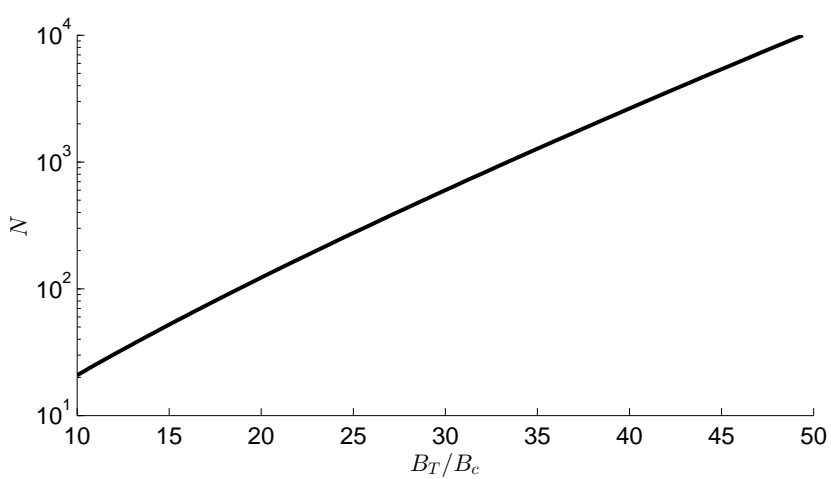

Figure 2. Equivalent number of independent realizations required in a CWdriven $\mathrm{RC}$ in order to ensure the same typical efficiency than a TR-driven one, as given by (27).

where $p_{K}(x)$ is the first derivative of $F_{K}(x)$ (to be computed numerically), and $\alpha=C_{r} B_{T} \bar{W} \kappa^{2} / C_{i} B_{c}$. This pdf serves as a reference in sec. IV.

The mode, and also the mean of $\eta_{\mathrm{TR}}$ are well approximated as

$$
\mathrm{Mo}\left[\eta_{\mathrm{TR}}\right] \simeq\left\langle\eta_{\mathrm{TR}}\right\rangle \simeq \frac{C_{r}}{C_{i}} \frac{B_{T}}{B_{c}}\left\langle W\left(f_{c}\right)\right\rangle(\kappa / \mathrm{Mo}[K])^{2}
$$

where

$$
\mathrm{Mo}[K] \simeq a \ln ^{b}\left(4 B_{T} / B_{c}\right)
$$

was shown to be accurate to better than $1 \%$ in [8], when $a=0.749$ and $b=0.678$.

For a harmonic excitation, the mode is

$$
\mathrm{Mo}\left[\eta_{\mathrm{CW}}^{N}\right]=\left\langle\eta_{\mathrm{CW}}\right\rangle \ln N=\frac{C_{r}}{C_{i}}\left\langle W\left(f_{c}\right)\right\rangle \ln N .
$$

From (23) and (25), we can now express (20) as

$$
G_{p}^{N}=\frac{B_{T}}{B_{c}} \frac{\kappa^{2}}{\ln N}\left[a \ln ^{b}\left(4 B_{T} / B_{c}\right)\right]^{-2} .
$$

It could seem surprising, at first sight, to see that for $N=1$, $G_{p}$ is expected to be infinite. This is a direct consequence of the high probability of observing a weak field intensity for a single realization. In fact, the case $N=1$ is of no practical use, as in any experimental setup typically involves at least a few tens of independent stirrer states.

Eq. (26) provides a direct estimate of how many realizations (e.g., stirrer steps) should be carried out in case of an harmonic excitation, in order to obtain a performance similar to that expected with TR excitations. Enforcing $G_{p}^{N}=1$, one would need

$$
N=\exp \left\{\frac{B_{T}}{B_{c}}\left[a \ln ^{b}\left(4 B_{T} / B_{c}\right)\right]^{-2}\right\}
$$

independent realizations. A graphical representation of (27) in Fig. 2 shows that keeping up can quickly become unaffordable. Eq. (27) can also be interpreted as the ratio, between the bandwidth that should be spanned for CW frequency stirring, with respect to that needed by a TR signal in order to obtain the same efficiency. The underlying reason for this potentially huge difference in the performance is to be found in the collaborative nature of TR, where the $N$ degrees of freedom are used coherently at the same time, as opposed to the CW case, where they are used separately in a non-coherent manner.
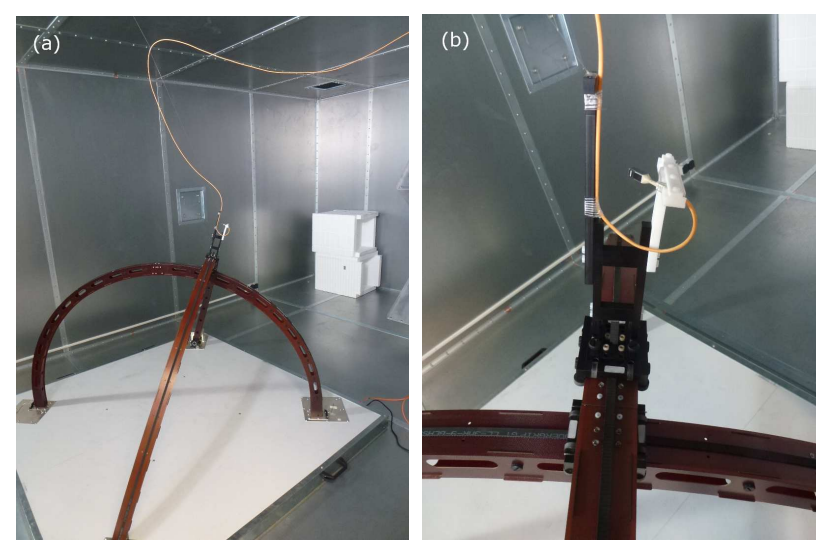

Figure 3. The hemispherical positioner (a) and the electro-optical probe (b) used during the experimental validation.

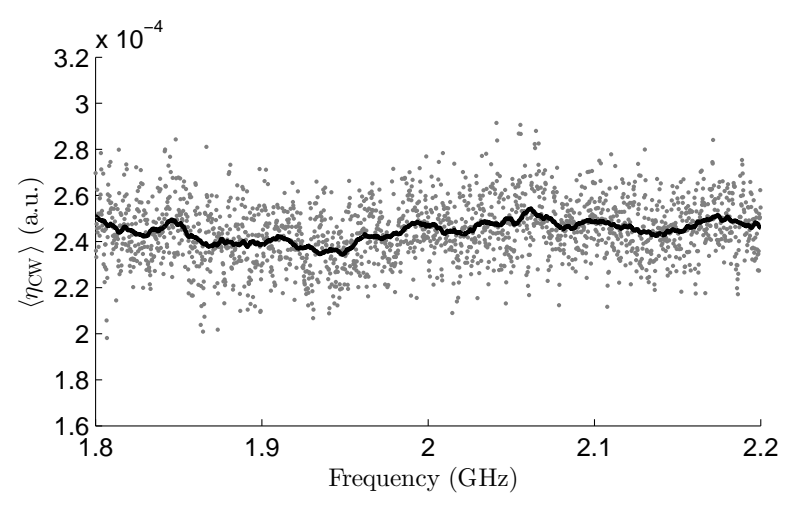

Figure 4. Estimate of $\left\langle\eta_{\mathrm{CW}}\right\rangle$ (grey dots) and its smoothed version (black line) obtained with a moving average over a $20 \mathrm{MHz}$ bandwidth.

\section{EXPERIMENTAL VALIDATION}

One of Supelec's RCs was used for the validation; its dimensions are $6.6 \times 3.0 \times 2.5 \mathrm{~m}^{3}$ with an expected lowest usable frequency around $400 \mathrm{MHz}$. We did not study $\eta_{\mathrm{CW}}^{N}$ experimentally, since two decades of research on RCs have thoroughly validated the good accuracy of (19) when using harmonic excitations [13], [14]. It is sufficient to estimate $\langle W(\nu)\rangle$, as discussed in sec. II. The validation therefore focused on the performance of TR excitation and the power gain, so that we did not apply any stirring technique, as TR is based on self-averaging. The presence of the stirrer was still useful for breaking symmetries in the cavity, as well as for reducing any direct illumination of the receiver from the source antenna.

A monocone antenna, mounted between a corner of the RC and the stirrer, was used for the excitation of the RC. Transfer functions between this antenna and a phase-sensitive electrooptical probe (Enprobe EFS-105) were measured by means of a vector network analyzer, collecting data over 5000 frequency samples in the bandwidth $[1.8,2.2] \mathrm{GHz}$. The probe was made to scan a hemispherical surface with a radius of $1 \mathrm{~m}$, thanks to the low-perturbation robot depicted in Fig. 3, together with the probe. The residual field coherence, or correlation, as the robot moves was estimated to be about $80 \%$, thanks to its thin 

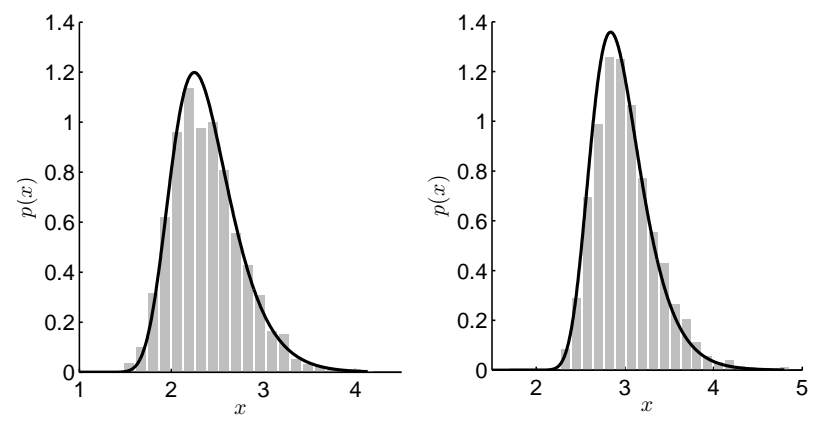

Figure 5. Empirical and theoretical pdfs of $\left\|x_{\mathrm{TR}}(t)\right\|_{\infty} / A_{o}$, for $B_{T}=32$ $\mathrm{MHz}$ (left) and $256 \mathrm{MHz}$ (right).

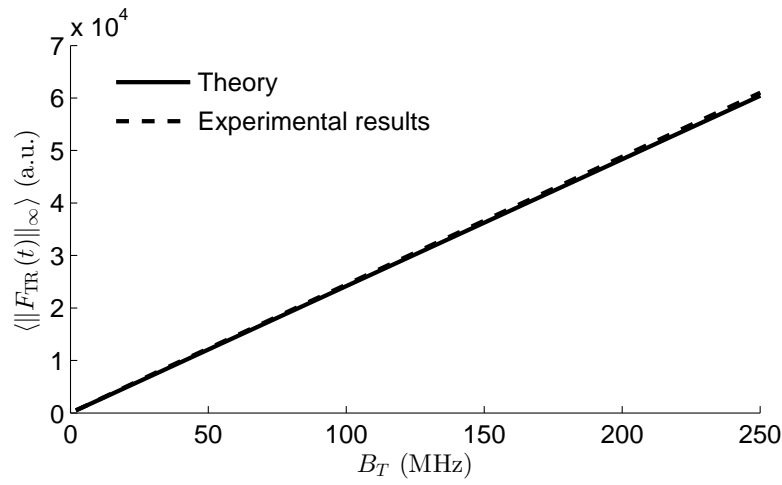

Figure 6. Estimate of $\left\langle\left\|f_{\mathrm{TR}}(t)\right\|_{\infty}\right\rangle$ as a function of $B_{T}$.

structure made out of glass fibers immersed in a plastic matrix. 3431 positions were thus considered, uniformly distributed with an average distance of $3.75 \mathrm{~cm}$, i.e., $\lambda / 4$ at $2 \mathrm{GHz}$.

From this set of data we first computed $\eta_{\mathrm{CW}}$, shown in Fig. 4 to be indeed slowly varying. Field-related results were not corrected for the antenna factor of the probe, since our models refer to ratios of efficiencies affected by the same constants. Therefore efficiencies are all reported in arbitrary units (a.u.).

Time-domain responses $\phi(t)$ of the RC were computed at each position, for several bandwidths $B_{T}$, up to $256 \mathrm{MHz}$. It was thus possible to estimate a time constant $\tau=2.14 \mu \mathrm{s}$ by means of a least-square fitting of $\left\langle\phi^{2}(t)\right\rangle ; \tau$ is needed in order to compute the coherence bandwidth $B_{c}=467 \mathrm{kHz}$ required by our models.

Having direct access to the impulse responses, TR excitations can be defined as in (4), and their actual peak values recorded; all the results about TR excitations involve $G(\nu)=1$ over $B_{T}$, i.e., a sine cardinale (sinc) pulse in the time domain. The histogram of the peak values, normalized to $A_{o}=\bar{W} B_{c} B_{T}$ (see (15)), is shown in Fig. 5, together with the theoretical pdf of the overshoot factor $K$, obtained by numerically differentiation of (11). These results refer to two bandwidths, $B_{T}=32$ and $256 \mathrm{MHz}$, corresponding to fractional bandwidths $B_{T} / f_{c}$ equal to $1.6 \%$ and $12.8 \%$, respectively. Both distributions present a good agreement, while their mode passes from 2.25 to 2.85 , i.e., slightly more than a $25 \%$ change for an eightfold increase in the excitation
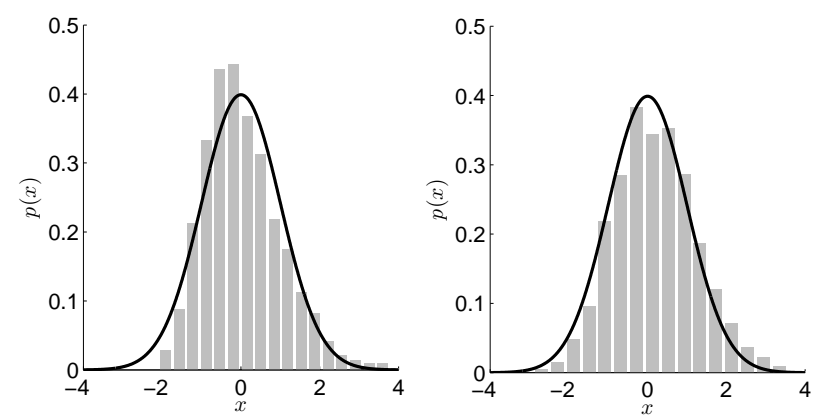

Figure 7. Empirical and theoretical pdfs of $\left(\left\|f_{\mathrm{TR}}(t)\right\|_{\infty}-u\right) / \sqrt{u}$, with $u=\left(B_{T} / B_{c}\right) \bar{W}$, for $B_{T}=2 \mathrm{MHz}$ (left) and $32 \mathrm{MHz}$ (right).

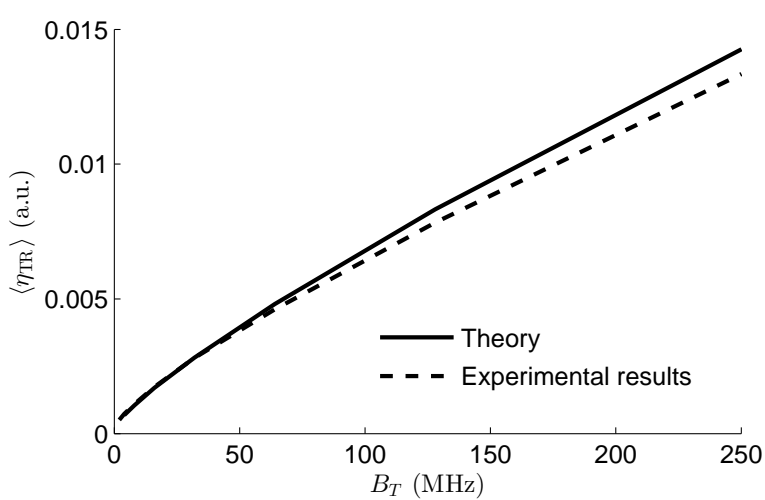

Figure 8. Estimate of $\left\langle\eta_{\mathrm{TR}}\right\rangle$ as a function of $B_{T}$.

bandwidth.

Conversely, the output field generated by these excitations is expected to be proportional to the bandwidth, as stated by (9). Fig. 6 supports this prediction for the mean value of TR output fields. In order to assess whether their mean value is a good representative of TR-generated fields, Fig. 7 presents the histograms obtained by computing $\left(\left\|f_{\mathrm{TR}}(t)\right\|_{\infty}-u\right) / \sqrt{u}$, with $u=\left(B_{T} / B_{c}\right) \bar{W}$. If TR-generated fields were normally distributed according to the central-limit theorem, the previous operation should lead to a random variable well described by a standard normal distribution. The results in Fig. 7 confirms that this model is consistent for $B_{T}=32 \mathrm{MHz}$, while for $B_{T}=2$ $\mathrm{MHz}$ the approximation, while not perfect, is still reasonable. This last observation is surprising, since for $B_{T}=2 \mathrm{MHz}$, the input signal just covers slightly more than 4 coherence bandwidths, i.e., a very low number of degrees of freedom. These results have a strong effect on the confidence margin of TR-generated fields, as discussed in the next section.

The TR efficiency was then assessed, first for its mean value shown in Fig. 8. Its sub-linear dependence on $B_{T}$ is a direct consequence of how the maxima of RC impulse responses increase, although slowly, with $B_{T}$ [8]. The slight discrepancy (about $6 \%$ ) between theory and experiments could seem surprising, since previous results were in very good agreement for both the numerator and the denominator of $\eta_{\mathrm{TR}}$. In fact, predicting the pdf of the ratio of random variables is not a simple matter, in particular in the case of interest, 

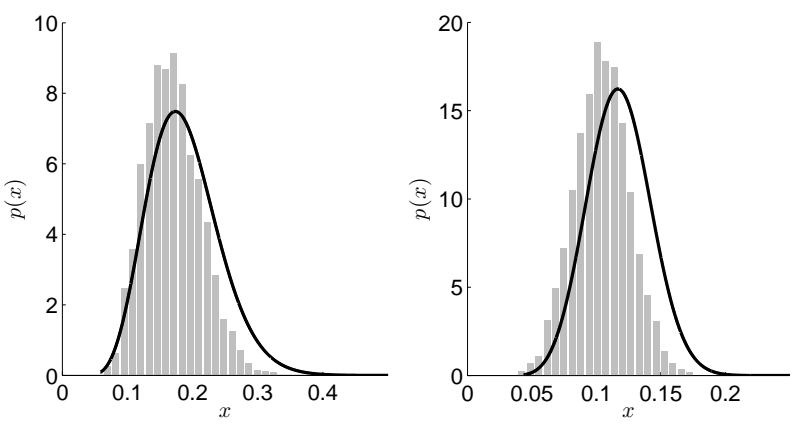

Figure 9. Empirical pdfs of $\eta_{\mathrm{TR}} /\left(B_{T} \bar{W} / B_{c}\right)$ and approximate pdf obtained by assuming a deterministic output, as defined in (22) for: $B_{T}=32 \mathrm{MHz}$ (left) and $B_{T}=256 \mathrm{MHz}$ (right).

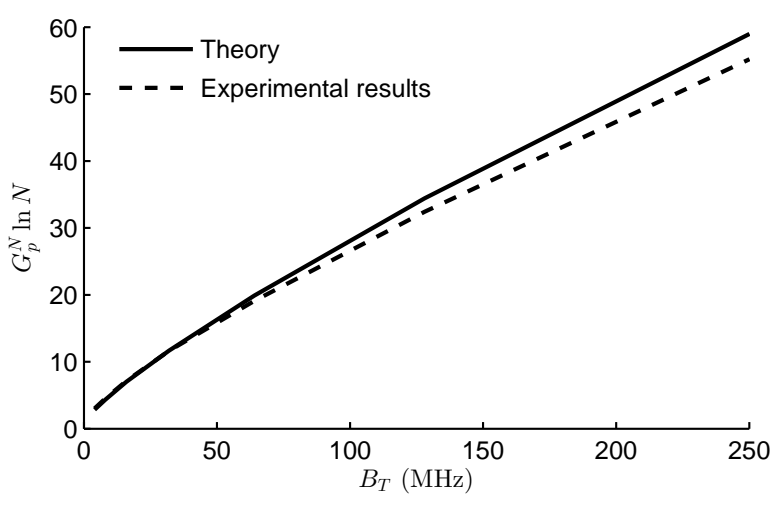

Figure 10. Normalized gain $G_{p}^{N} \ln N$ as a function of $B_{T}$

since the numerator and denominator are not independent, having a common origin in the impulse responses of the RC. Having chosen to approximate the field generated by $\mathrm{TR}$ as a deterministic quantity is the reason for this residual error, which is confirmed in the comparison of the empirical and theoretical probability distributions (22) in Fig. 9.

Finally, we present data confirming the validity of (26), shown in Fig. 10. Attention should be paid to the meaning of $G_{p}^{N} \ln N$ : it does not represent the final power gain, but a normalized version independent of $N$. The slight disagreement is a direct consequence of the one in the modeling of $\eta_{\mathrm{TR}}$.

\section{CONFIDENCE INTERVALS}

The good agreement of experimentally estimated TRgenerated peak fields with a standard Gaussian distribution implies that indeed output fields do converge as dictated by the central-limit theorem, i.e., they span a confidence interval $C_{\mathrm{TR}}$, e.g., with a $95 \%$ probability, approximately equal to

$$
C_{\mathrm{TR}} /\left\langle\eta_{\mathrm{TR}}\right\rangle=2 \sqrt{B_{c} / B_{T}},
$$

to be compared with the one obtained for $\eta_{\mathrm{CW}}^{N}$

$$
C_{\mathrm{CW}}^{N} /\left\langle\eta_{\mathrm{CW}}^{N}\right\rangle=\ln \left[\frac{1-\sqrt[N]{0.025}}{1-\sqrt[N]{0.975}}\right] / \ln N .
$$

These confidence intervals, represented as functions of the available number of degrees of freedom, are shown in Fig. 11.

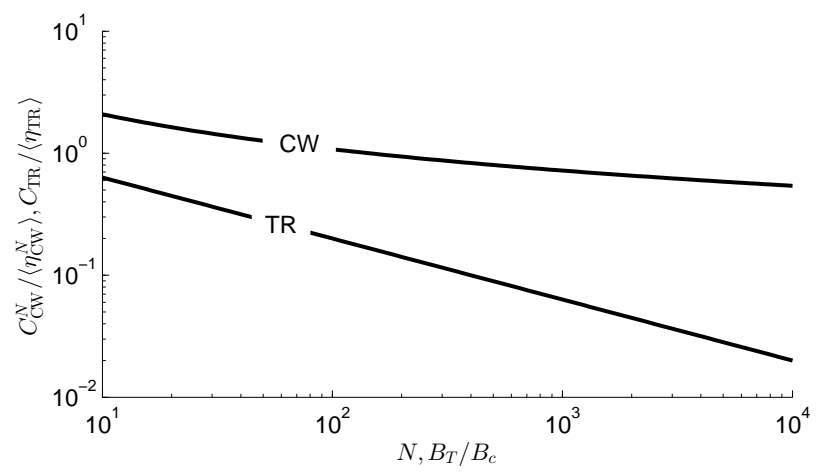

Figure 11. Relative confidence intervals of the conversion efficiencies for a $95 \%$ probability, as a function of the number of degrees of freedom.

The self-averaging property of TR excitations is the inherent cause for its faster convergence.

It could therefore be disappointing to have $\eta_{\mathrm{TR}}$ still featuring a pdf that does not appear to converge to a deterministic value. In fact, the reasons for the statistical dispersion of $\eta_{\mathrm{TR}}$ are utterly different from those that are behind $\eta_{\mathrm{CW}}$. In the case of TR, the output field does converge to a deterministic value, as demonstrated in the previous section in Fig. 7; but when normalized to the input peak power, it is the stronger dispersion of this last quantity that increases the spread of $\eta_{\mathrm{TR}}$.

Conversely, in the case of $\mathrm{CW}$ excitations, the statistical dispersion only depends on the output fields, meaning that its actual value is hardly predictable. Therefore, in the case of TR excitations, a non deterministic $\eta_{\mathrm{TR}}$ has a simple meaning: while the output field is predictable with a high degree of accuracy, the actual amount of peak input power is not. As such, it could require more, or less, peak power than expected on average, but this uncertainty will hardly have any major effect on the field intensity.

\section{CONCLUSIONS}

The benefits of driving an RC with TR signals has been confirmed on three levels: 1) a higher conversion efficiency, 2) a reduced uncertainty in the actual field level, and this 3) without the need for multiple realizations (stirring). The increase in the efficiency may well be translated into a lightening in the peak-instantaneous power specifications for power amplifiers.

Our work has also proven that TR does not necessarily rhyme with wide-band signals, as acoustical TR has got us used to. Narrow bands can be envisaged as TR excitation signals, since the only constraint is $B_{T} / B_{c} \gg \pi / Q$; with RCs easily displaying $Q \gtrsim 1000$, it should be possible to keep using standard power amplifiers while reaping TR benefits. Furthermore, a minimum frequency resolution can also be preserved.

The price to pay is the need for an overhaul in the use of $\mathrm{RCs}$, as TR excitations require thinking in terms of spatiallyresolved stresses. A TR-driven RC works by focusing part of the injected energy over a reduced region of space during 
a short time interval. As a result, tests would need to be repeated when switching from one position to the next one, not unlike an anechoic chamber, where directions are tested one after the other. Depending on the size of the focal spot, and ultimately, on the desired spatial resolution and the EUT dimensions, the number of excitations required could vary wildly. Predicting this number is tricky, as a large number of potential implementations for TR-powered RC facilities can be devised. A discussion about the benefits and shortcomings of the various classes of TR implementations can be found in [6], where a special attention was given to the use of TRexcitations for test facilities.

\section{REFERENCES}

[1] C. Draeger, J.-C. Aime, and M. Fink, "One-channel time-reversal in chaotic cavities: Experimental results," The Journal of the Acoustical Society of America, vol. 105, no. 2, pp. 618-625, 1999.

[2] G. Lerosey, J. de Rosny, A. Tourin, A. Derode, G. Montaldo, and M. Fink, "Time reversal of electromagnetic waves," Phys. Rev. Lett., vol. 92, May 2004.

[3] A. Cozza, "Statistics of the performance of time reversal in a lossy reverberating medium," Physical Review E, vol. 80, no. 5, p. 056604 , 2009.

[4] A. Cozza and H. Moussa, "Enforcing deterministic polarisation in a reverberating environment," Electronics Letters, vol. 45, no. 25, pp. 1299-1301, 2009.

[5] A. Cozza, "Increasing peak-field generation efficiency of reverberation chamber," Electronics Letters, vol. 46, no. 1, pp. 38-39, January 2010.

[6] _ "Emulating an anechoic environment in a wave-diffusive medium through an extended time-reversal approach," IEEE Transactions on Antennas and Propagation, vol. 60, no. 8, pp. 3838-3852, 2012.

[7] R. E. Richardson, Reverberant Microwave Propagation, ser. Technical Report 2000-2008. Naval Surface Warfare Center Dahlgreen Div VA, Oct. 2008.

[8] A. Cozza and F. Monsef, "Stochastic Modeling of Impulse Responses Observed in Reverberating Environments," to be submitted to IEEE Transactions on Vehicular Technology, 2015.

[9] — " "Multiple-Source Time-Reversal Transmissions in Random Media," IEEE Transactions on Antennas and Propagation, vol. 62, no. 8, pp. 4269-4281, May 2013.

[10] A. Cozza, "The role of losses in the definition of the overmoded condition for reverberation chambers and their statistics," IEEE Transactions on Electromagnetic Compatibility, vol. 53, no. 2, pp. 296-307, 2011.

[11] T. H. Lehman, "A statistical theory of electromagnetic fields in complex cavities," Interaction Notes, Note, vol. 494, 1993.

[12] F. Monsef and A. Cozza, "Variability and confidence intervals of the power measured in a reverberation chamber," IEEE Transactions on Electromagnetic Compatibility, vol. 56, no. 5, pp. 1238-1241, Oct 2014.

[13] T. Lehman and G. Freyer, "Characterization of the maximum test level in a reverberation chamber," in IEEE International Symposium on Electromagnetic Compatibility, Aug 1997, pp. 44-47.

[14] J. Ladbury, G. Koepke, and D. Camel, "Evaluation of the NASA Langley Research Center mode-stirred chamber facility," NIST technical note, no. 1508, pp. 1-282, 1999.

[15] M. Hoijer, "Maximum power available to stress onto the critical component in the equipment under test when performing a radiated susceptibility test in the reverberation chamber," IEEE Transactions on Electromagnetic Compatibility, vol. 48, no. 2, pp. 372-384, 2006.

[16] A. Derode, A. Tourin, and M. Fink, "Random multiple scattering of ultrasound. ii. Is time reversal a self-averaging process?" Phys. Rev. E, vol. 64, p. 036606, Aug 2001. 